\title{
Age-related Spike Timing Dependent Plasticity of Brain-inspired Model of Visual Information Processing with Reinforcement Learning
}

\author{
Petia Koprinkova-Hristova \\ Institute of Information and Communication Technologies, \\ Bulgarian Academy of Sciences \\ Sofia, Bulgaria \\ Email: pkoprinkova@bas.bg
}

\author{
Nadejda Bocheva \\ Institute of Neurobiology, \\ Bulgarian Academy of Sciences \\ Sofia, Bulgaria \\ Email: nadya@percept.bas.bg
}

\begin{abstract}
The paper summarizes our efforts to develop a spike timing neural network model of dynamic visual information processing and decision making inspired by the available knowledge about how the human brain performs this complicated task. It consists of multiple layers with functionality corresponding to the main visual information processing structures starting from the early level of the visual system up to the areas responsible for decision making based on accumulated sensory evidence as well as the basal ganglia modulation due to the feedback from the environment. In the present work, we investigated age-related changes in the spike timing dependent plastic synapses of the model as a result of reinforcement learning.
\end{abstract}

\section{INTRODUCTION}

$\mathbf{F}$ OR centuries scientists were trying to discover the way we learn how to behave in an unknown environment without prior information about which the proper actions are. Every day humans make a large number of decisions based on sensory information as well as on previously accumulated experience. In decision making based only on sensory information the response is determined by the stimulus characteristics. However, the pioneering work of Pavlov on conditional and unconditional reflexes of living creatures revealed yet another intriguing characteristics of the natural intelligence: the ability to plane its future behaviour not only based on its current sensory information but also on its past experience accumulated via trial and error thus accounting for its past actions outcome in similarly sensed environment. It gives rise to development of theory of reinforcement learning starting with the seminal work of [1] and their actor-critic architecture that was able to learn from simple punish/reward feedback from the environment. It can be considered as one of the first artificial systems possessing a kind of artificial intelligence. Parallel to the research based on behavioral experiments neurologists tried to discover the brain counterparts of sensory information processing as well as of the reinforcement learning.

The visual system of human brain was probably the most investigated by neurobiologists. The hierarchical processing

This work is supported by the Bulgarian Science Fund project No DN02/3/2016 "Modelling of voluntary saccadic eye movements during decision making". structures starting from our light sensors - the eyes - through optic nerve to visual cortex were well established. It also has been shown that several brain areas like lateral intraparietal area (LIP) accumulate evidence supporting the alternative decisions ([2]) and transform eye sensory information in a decision variable that directs action. Many models assume that a choice is made when the accumulated evidence for one of the sensory signals reaches a predefined value. It has been shown (e.g.[3]) that the basal ganglia can modulate this threshold level. Other studies (e. g. [4]) imply that the basal ganglia could also modify the rate of sensory evidence accumulation. These results suggest an important role of the basal ganglia in perceptual decision making. Existing evidence (e.g. [5], [6], [7]) suggests also a significant role of the basal ganglia on learning by trial and error to acquire a reward, i.e. reinforcement learning. The role of the basal ganglia in reinforcement learning is related to the differential responses of the dopaminergic neurons in one structure of the basal ganglia (substantia nigra compacta) to unexpected and predicted rewards and to the omission of an expected reward. Recently, several modeling attempts (e.g.[8], [9]) try to integrate these two functions of the basal ganglia - in decision making processes and in reinforcement learning in a common framework starting from the cortical input.

In contrast to these models, in previous work [10] we developed a spike timing neural network model that includes the major structures related to dynamic visual information processing i.e. including the structures that provide the sensory information for making a decision starting from the retinal input. The parallel structure of the model was adopted from [11] while the basal ganglia connectivity was adopted from [12]. Main advances in our work in comparison to these two previous works were as follows:

- While in [12] the model consisted of cellular network structures whose neurons are modelled by firing rate equations, in our model we used spike timing neurons organized in layers with the same connectivity.

- In [12] dopamine signal is calculated as temporal differ- 
ence error that was directly exploited to adjust dopamine synapses. In our model we have an additional layer of neurons whose spiking activity was equivalent to dopamine release into corresponding synapses having spike timing dependent plasticity.

- In [12] the layer responsible for visual information processing and generating sensory input to the basal ganglia is simplified and in [11] it is completely missing while our model includes multiple layers corresponding to hierarchical brain structures performing dynamic visual information processing.

- In contrast to [11] where lateral connections within layers are limited, our model has much more elaborated connectivity similar to that proposed in [12].

These characteristics of our model made it more realistic and provide greater opportunities for understanding the process of learning and decision making in human brain. [13].

The model was implemented using NEST 2.12.0 simulator

Further attempts to improve our model were as follows:

- Enhanced connectivity of visual information processing layers with multiple feedback connections and spike timing dependent plasticity in its synapses reported in [14].

- Enhanced feedback connectivity from basal ganglia back to visual cortex.

The present paper summarizes our model structure and connectivity and investigates age-related changes of its dynamic synapses induced by age-specific external reinforcement. It is organized as follows: Section II briefly describes the model structure; next simulation experiment with moving dot stimulus and external age-related reinforcement signal was presented and achieved after training values of spike timing dependent plastic (STDP) synapses were presented and commented; the paper finishes with concluding remarks and directions for future work.

\section{Model Structure}

Based on the available data about human brain structures playing role in visual motion information processing and decision making, as well as their connectivity, the hierarchical model proposed in [15] consists of two basic substructures: related to visual information perception and sensory-based decision making and the basal ganglia and their function on the perceptual decision via external reinforcement.

Each layer consists of neurons positioned in a regular twodimensional grid. The receptive field (area of neurons from a given layer that are connected to a given neuron from the same or neighbour layer) of each individual neuron depends on the function of the layer it belongs to as well as on its position inside the layer. The neurons' dynamical models as well as intra- and inter-layer connectivity are described in consecutive subsections.

\section{A. Visual information perception and sensory-based decision}

The structure of perceptual layers up to LIP area involved in the sensory-based decision making reported in [15], [16], [17] is shown in Fig. 1. It consists of the following layers: Retinal ganglion cells (RGC); Lateral geniculate nucleus (LGN); Thalamic reticulate nucleus (TRN) and Interneurons (IN); Primary visual cortex (V1);Middle temporal (MT) area; Medial superior temporal (MST) area and Lateral intraparietal cortex (LIP).

Following the commonly accepted models from [18], [19], the reaction of retinal ganglion cells to luminosity changes was simulated by a spatiotemporal filter whose spatial component has circular shape modelled by a difference of two Gaussians and the temporal component has a bi-phasic profile determined by difference of two Gamma functions. The continuous signal generated by convolution of this spatiotemporal kernel with the visual stimuli (images falling on the retina) is the electrical output current of retinal cells. Each retinal ganglion cell generates input current and is connected to its corresponding LGN neuron.

The structure of LGN layer is the same as that of the retinal layer. We have two layers of retinal cells and their corresponding LGN neurons, having identical positions of "oncenter off-surround" (ON) and "off-center on-surround" (OFF) cells placed in reverse order. Their positions are relative to the visual scene. For the LGN neurons we used the proposed in [20] model whose parameters were determined from in-vivo experiments. This layer sends forward signals to the next layer (V1) and receives excitatory feedback from it directly as well as via inhibitory connections through interneurons and TRN.

The structure of thalamic relay that prepossesses the feedback from the visual cortex (V1) to LGN has structure adopted from [21] as shown on Fig. 1. The interneurons receive excitatory inputs from both retinal neurons (feedforward) and primary visual cortex (feedback) and send inhibitory signal to their corresponding LGN neuron. The TRN neurons mediate excitatory feedback from visual cortex and send another inhibitory input to the corresponding LGN neurons. Since we have a TRN and an interneuron attached to each LGN neuron, their positions coincide on the LGN grid of neuron positions. For simplicity, in our model the feedback connectivity from V1 was the same as the feedforward connectivity from LGN to V1. In [16] the presence and strength of such feedback connectivity on the spiking activity of the primary visual cortex was investigated by simulations. It was demonstrated that it has modulatory effect on the selectivity of V1 neurons.

As in [19], the neurons in V1 layer are separated into four groups - two exciting and two inhibiting populations connected via corresponding excitatory and inhibitory intra-layer (lateral) connections. According to [19] and [18] the ratio of exiting to inhibiting neurons should be $4 / 1$. All neurons are positioned at the same two-dimensional space and the inhibiting neurons are dispersed among bigger groups of exciting neurons. Since the neurons in V1 layer are orientation sensitive, they have elongated receptive fields defined by a Gabor probability function 


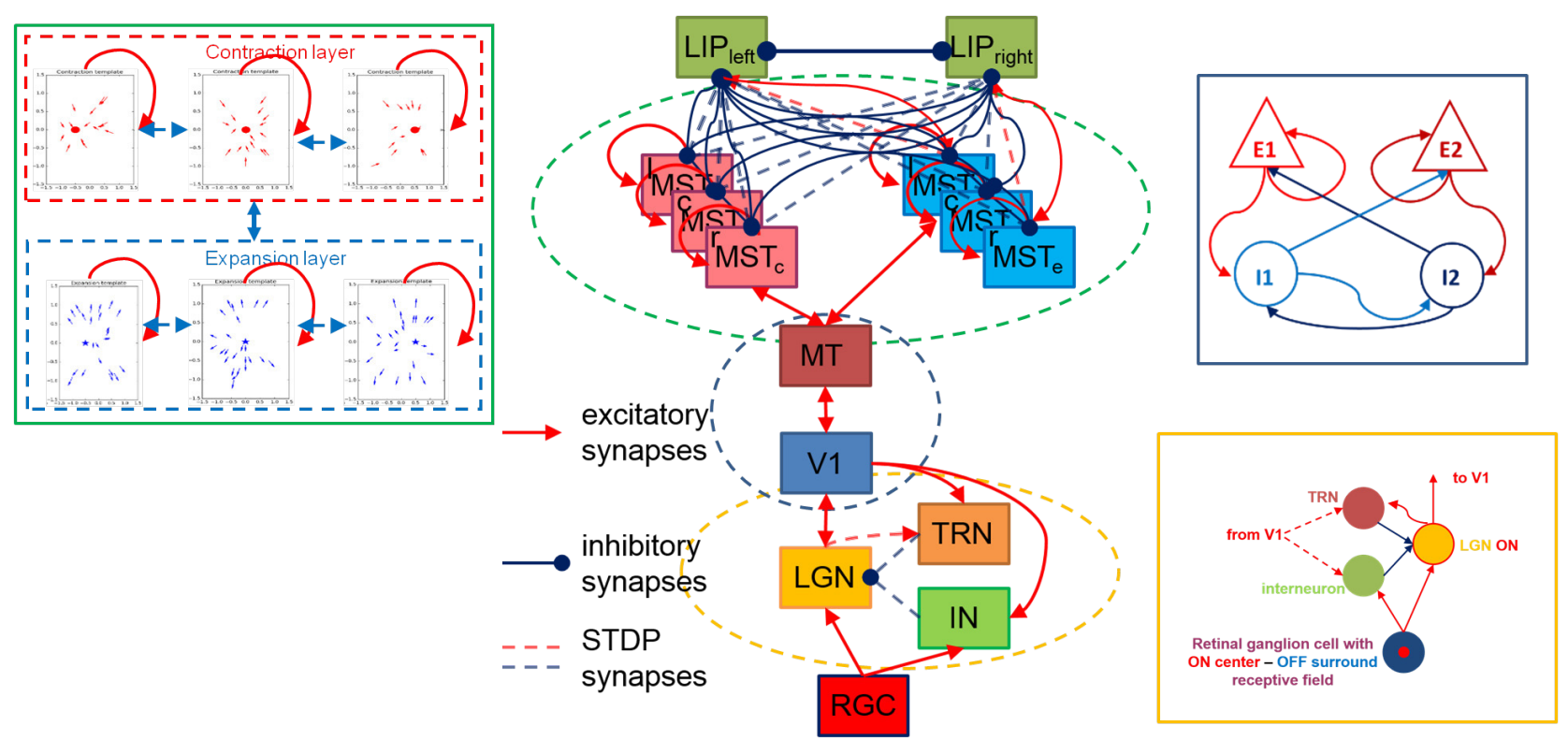

Fig. 1. Model of dynamic sensory information processing. Each box represents a two-dimensional layer of neurons. The color of connections corresponds to their type, i.e. red for excitatory and blue for inhibitory ones.

with orientation and phase parameters like in [22]. The 2D maps containing the neurons' orientations and phases at corresponding 2D grid of the V1 layer should have typical for the mammalian brain "pinwheel-structure". Among the proposed approaches for artificial design of such a structure, that of [23] is relatively new and easily implemented one. That is why we used it to design V1 orientation and phase maps of our model ([17]). The absolute values of lateral connection weights in V1 are determined on the basis of neuronal Gabor correlations with respect to their positions, phases and orientations. The sign of a connection weight depends on whether it is excitatory (positive) or inhibitory (negative). Besides, as in [19], neurons from inhibitory populations connect preferentially to neurons having a receptive field phase difference of about $180^{\circ}$. In our model, we defined the spatial frequencies and standard deviations of the Gabor filters for lateral connection weights so as to obtain approximately circular receptive fields for all neurons in the layer.

The next (MT) layer is the major motion information processing structure and it has identical structure to V1 layer. The lateral connections are designed in the same way while the connections from V1 cells depend on the angle between orientation preferences of each pair of cells according to [24]. The orientation and phase maps of this layer were generated in the same way as in the case of V1 layer.

The following Medial Superior Temporal Area (MST) was modeled like in [25] by two layers sensitive to expansion and contraction movement patterns that occur during the selfmotion of the observer . Each MST cell has assigned contraction/expansion pattern template having circular shape and focal point at MT layer. Following [25], the MST neurons have on-center receptive fields. Each MST neuron collects inputs from MT cells corresponding to its pattern template. Both layers have intra- and interlayer excitatory/inhibitory recurrent connections between cells having similar/different sensitivity (see Fig. 1). These lateral connections are determined based on neurons' positions and template similarities. All neurons have Gaussian receptive fields. Connections within expansion/contraction layers are excitatory or inhibitory in dependence on their focal points' similarity. Connections between expansion and contraction layers are all inhibitory and depend both on similarities of their positions and focal points.

LIP area is the last layer of perceptual part of the model that is responsible for making decisions based on accumulated sensory evidence. Since our model aims to decide whether the expansion center of moving dot stimulus is left or right from the stimulus center, in [15] we proposed a taskdependent design of excitatory/inhibitory connections from MST expansion/contraction layers to the two LIP sub-regions whose increased firing rate corresponds to either of two motor responses - eye movement to the left or right. Both LIP areas are connected via excitatory connections to neurons in MST expansion layer having template focal points (left or right) corresponding to their motor responses (left or right). The rest of neurons are connected via inhibitory connections. There are also lateral inhibitory connections between both groups of LIP neurons.

\section{B. Basal Ganglia}

In order to modulate LIP decisions using external reinforcement signal, its output (considered as processed and accumulated sensory information) was further fed into a group of subcortical nuclei - Basal ganglia (BG). These include Striatum, Globus Pallidus externa (GPe), Subthalamic Nucleus 
(STN), Substantia Nigra pars reticulata $(\mathrm{SNr})$ and Substantia Nigra pars compacta (SNc) [12], [11]. The structure of BG in our model, shown on Fig. 2 combines ideas from both [12] and [11].We excluded the internal segment of the Globus Pallidis (GPi) from the model as it is an output of the Basal ganglia to the thalamus, while we are interested in the effects of BG activity on eye movement control. For this reason, we considered only the other output structure of the Basal ganglia - the $\mathrm{SNr}$ as it projects to the superior colliculus (SC), a structure controlling saccade generation.

Like in [12], our model incorporates layers of Striatum, GPe/STN structure and SNr. However, it consists of two parallel structures, receiving inputs from the left and right saccade selecting LIP areas respectively. These two channels (left and right) are connected via mutually inhibiting connections through their GPe areas like in [11]. Additionally, in contrast to [12], our model has a complete 2D layer of neurons producing dopamine neuromodulator $(\mathrm{SNc})$ and dopaminedependent synapses.

Striatum is divided into two sub-areas depending on the type of dopamine receptors they express (D1 and D2 on Fig. 2). Both are modelled as 2D layers of integrate and fire (IAF) neurons whose lateral connections have short-range excitation and long-range inhibition characteristics like in [12]. These two sub-areas form the inputs to the direct and indirect pathways that process signals through the basal ganglia. The cortical input (coming from the LIP layer) has dopaminergic synapses whose weights were randomly initialized and they are dynamically changed in dependence on the spiking activity of $\mathrm{SNc}$ area (considered as dopamine secreting structure). The NEST simulator offers dopaminergic synapse model from [26]. Since our model includes also anti-dopamine synapses (from LIP to D2 sub-area of the Striatum) whose dynamics has to be opposite to that of dopaminergic ones, we've modified the model from [26] by converting the amplitudes $A_{+}$and $A_{-}$of the dopamine eligibility trace dynamics from positive to negative.

The Globus Pallidus externa (GPe) and Subtalamic Nucleus (STN) pairs consists of 2D grid of pairs of neurons connected one-to-one via glutamatergic (excitatory) and GABAergic (inhibitory) connections as shown on Fig. 2. The GPe layer has also lateral connections having negative center and positive surround shape as in [12]. The structure receives inhibitory input from the second part of the Striatum (D2) via GPe and sends its output through STN via dopamine-dependent synapses to $\mathrm{SNr}$ (so called indirect pathway from Striatum to the BG output layer).

$\mathrm{SNr}$ was modelled by a 2D layer having short-range excitatory and long-range inhibitory lateral connections like both Striatum layers. Its input comes from both D1 layer of the Striatum (direct pathway) and GPe/STN structure (undirect pathway) via dopamine dependent synapses. SNr generates BG output to the motor-reaction controlling structure (SC).

$\mathrm{SNc}$ is considered as the brain area producing the neuromodulator dopamine in dependence on external motivation (reinforcement) input signal. In contrast to [12], where the dopamine level is calculated using temporal difference error, here we incorporated another 2D layer of neurons. The input to $\mathrm{SNc}$, coming from D1 area of the Striatum, was considered as the value function estimation like in [12]. Thus in order to "produce" the dopamine (temporal difference error) at the output of SNc, we set its inputs to be the value function for two consecutive time steps and the reinforcement signal as follows:

$$
\begin{array}{r}
V(t)=\mathrm{D} 1(t) \\
\delta(t)=\mathrm{r}(\mathrm{t})+\gamma V(t+1)-V(t) \\
\mathrm{SNc}=F(\delta(t))
\end{array}
$$

i.e. the dopamine release from the $\mathrm{SNc}$ is a function $F$ of the temporal difference error $\delta$ as in [5]. Here reinforcement signal $r(t)$ is external input current to the neurons in the $\mathrm{SNc}$ area $\left(r_{\text {left }}\right.$ and $r_{\text {right }}$ respectively) and the value function $V$ is associated with spiking activity in the D1 part of the Striatum. The discount factor $\gamma$ was set to 0.9 .

Since the SNc has the role of the critic within the model, its input connections from the Striatum were modelled as dopamine dependent synapses too.

Finally, the motor controlling structure SC was modelled by $2 \mathrm{D}$ layer of neurons receiving inputs directly from the LIP area (decision according to accumulated sensory information) as well as from the external reinforcement modulated output of $\mathrm{BG}$ (via $\mathrm{SNr}$ ).

The overall model connectivity is also enhanced by excitatory feedback connections from SC to their corresponding D1 and D2 areas of the Stratum as well as to LIP areas following recently reported findings [27], [28], [29]. Moreover we introduced mutually inhibiting connections between the two SC groups.

\section{Simulation Results And Discussion}

The overall model structure was implemented in NEST [13] simulator. For the neurons in LGN layer conductance-based leaky integrate-and-fire neuron model as in [20] was adopted. For the rest of neurons leaky integrate-and-fire (IAF) model with exponential shaped post-synaptic currents according to [30] was used.

The adjustable parameters in presented simulation are the strengths of dopaminergic synapses that vary in dependence on spiking activity of both SNc layers as well as STDP synapses of the visual perception sub-structure. The reinforcement inputs $r_{\text {left }}$ and $r_{\text {right }}$ are both teaching signals that control the dopamine level. In contrast to our preliminary investigations, where both reinforcement signals were constant generating currents of both $\mathrm{SNc}$ structures, here they were proportional to the difference between desired SC activity (generating current as in [14]).

The experiments with human test subjects separated into three age groups: 12 young persons (19-34 years old), 11 middle age (36-52 years old) and 12 elderly people (57-84 years old) were conducted and mean reaction time of each 


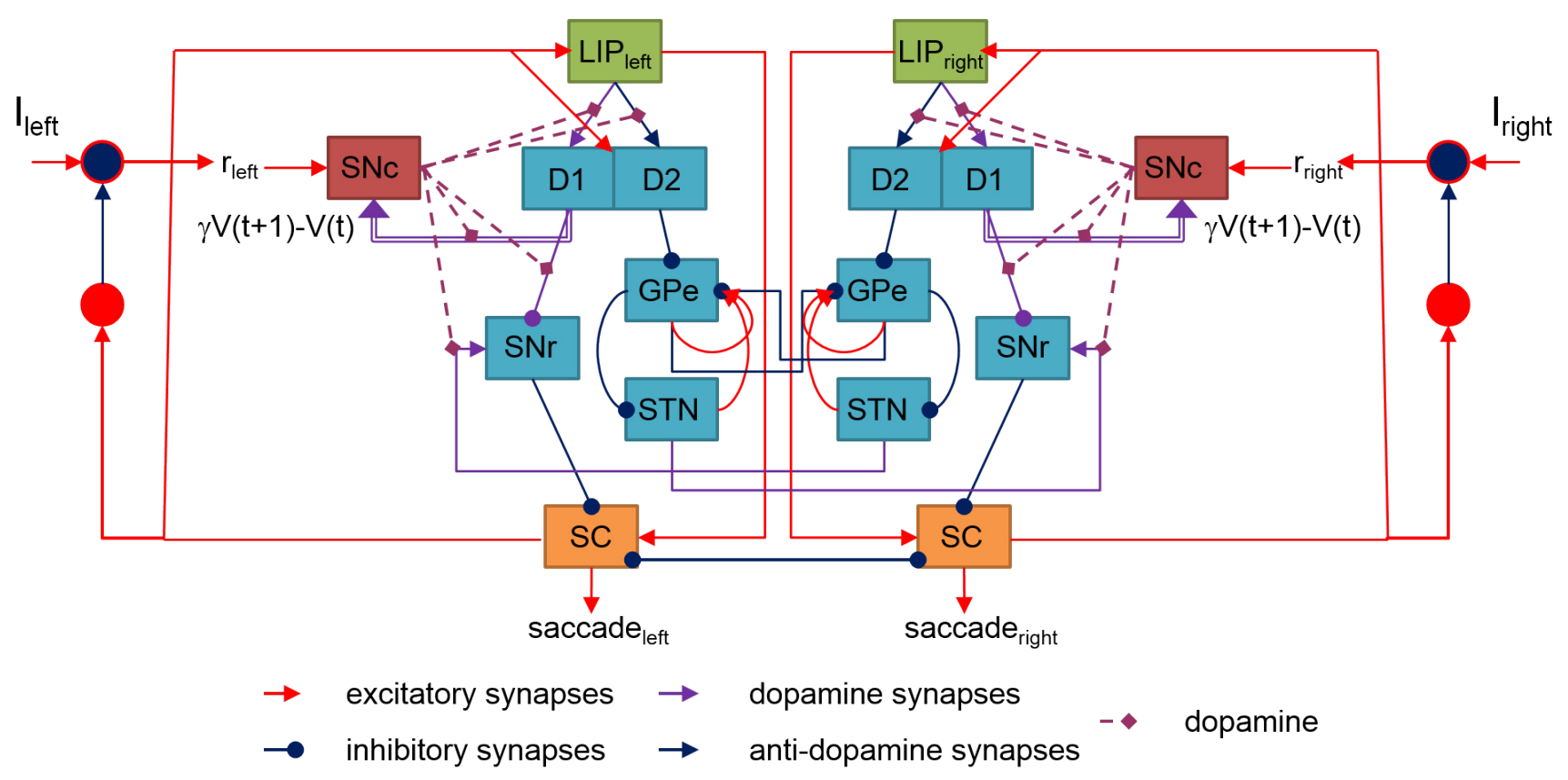

Fig. 2. Basal ganglia structure (in blue). It receives inputs from the decision-making area based on sensory information (LIP) as well as from the dopamine releasing area $(\mathrm{SNc})$ and generates activity biasing saccades generation via SC.

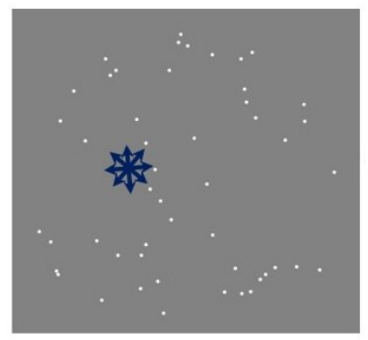

left

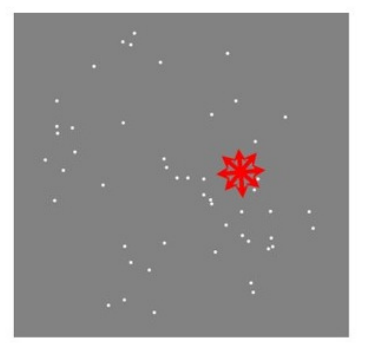

right
Fig. 3. Stimuli example.

age group was estimated [31]. The visual stimulation consists of projection of moving dot patterns on a computer screen. The test subjects are asked to indicate perceived expansion center that is left or right from the screen center as shown on Fig. 3. Details about experimental set-up were reported in [15].

In current investigation we simulated our model presenting as input moving dot stimuli with expansion center to the left of the screen center. As in [14] we've created training signals as generating currents $I_{\text {left }}$ and $I_{\text {right }}$ for the left and right LIP neurons respectively as follows:

$$
\mathrm{I}_{\text {left/right }}=A_{\text {left/right }} /\left(1+\exp \left(k_{\text {left/right }} t\right)\right)
$$

Amplitude $A_{\text {left/right }}$ defines maximal input current (in $p A)$ while $k_{\text {left/right }}$ determines settling time of the exponent that corresponds to the mean reaction time determined
TABLE I

MEAN AND VARIANCE OF WEIGHTS OF CONNECTIONS FROM MSTE TO LEFT LIP IN CASE OF REINFORCEMENT SIGNAL CORRESPONDING TO PERCEPTUAL DECISION (LEFT).

\begin{tabular}{|l|c|c|c|c|}
\hline Group & exc. mean & exc. var & inh. mean & inh. var \\
\hline Young & 3.63096 & 2.17431 & -5.82932 & 5.95606 \\
\hline Middle & 3.63059 & 2.17837 & -5.82684 & 5.95684 \\
\hline Elderly & 3.62601 & 2.17272 & -5.83082 & 5.95357 \\
\hline
\end{tabular}

TABLE II

MEAN AND VARIANCE OF WEIGHTS OF CONNECTIONS FROM MSTE TO RIGHT LIP IN CASE OF REINFORCEMENT SIGNAL CORRESPONDING TO PERCEPTUAL DECISION (LEFT).

\begin{tabular}{|l|c|c|c|c|}
\hline Group & exc. mean & exc. var & inh. mean & inh. var \\
\hline Young & 5.18551 & 5.03905 & -5.68507 & 5.79413 \\
\hline Middle & 5.21042 & 5.07128 & -5.68448 & 5.79909 \\
\hline Elderly & 5.19587 & 4.67182 & -5.70433 & 5.81697 \\
\hline
\end{tabular}

from experiments for each age group. For all three age groups amplitude values were the same: $A_{\text {left }}=200$ and $A_{\text {right }}=100$. In order to achieve approximately the settling time determined from experimental data, parameter $k_{\text {left } / \text { right }}$ has different values for three age groups (Y - young, M middle, $\mathrm{O}$ - old) with opposite signs for left and right case of stimulus respectively as follows: $k_{\text {left } / \text { right }}^{Y}=-/+0.02$; $k_{\text {left } / \text { right }}^{M}=-/+0.01 ; k_{\text {left } / \text { right }}^{O}=-/+0.005$.

We monitored the changes in STDP synapses of the model. Tables I - IV show the mean values and variances of connection weights between MSTe and LIP layers, while the 
TABLE III

MEAN AND VARIANCE OF WEIGHTS OF CONNECTIONS FROM MSTE TO LEFT LIP IN CASE OF REINFORCEMENT SIGNAL OPPOSITE TO PERCEPTUAL DECISION (LEFT).

\begin{tabular}{|l|c|c|c|c|}
\hline Group & exc. mean & exc. var & inh. mean & inh. var \\
\hline Young & 5.90917 & 5.82209 & -5.83044 & 5.95340 \\
\hline Middle & 5.90917 & 5.82209 & -5.82591 & 5.96218 \\
\hline Elderly & 5.90917 & 5.82209 & -5.83086 & 5.95266 \\
\hline
\end{tabular}

TABLE IV

MEAN AND VARIANCE OF WEIGHTS OF CONNECTIONS FROM MSTE TO RIGHT LIP IN CASE OF REINFORCEMENT SIGNAL OPPOSITE TO PERCEPTUAL DECISION (LEFT).

\begin{tabular}{|l|c|c|c|c|}
\hline Group & exc. mean & exc. var & inh. mean & inh. var \\
\hline Young & 3.64700 & 2.18479 & -5.95241 & 6.15924 \\
\hline Middle & 3.64970 & 2.18400 & -5.95241 & 6.15924 \\
\hline Elderly & 3.64957 & 2.18176 & -5.95241 & 6.15924 \\
\hline
\end{tabular}

Tables V - VIII show the mean values and variances of connection weights between four sub-structures within LGN (ON1, ON2, OFF1, and OFF2) and TRN obtained using external reinforcement signal for the three age groups with correct and reverse amplitude respectively. We did not observe changes in the inhibitory connections from MSTc area to both LIP left and right layers.

In case of reinforcement signal corresponding to perceptual decision both Tables I and II demonstrate tendency of decreased excitatiory connectivity and increased inhibitory connectivity with aging for both LIP areas. At the same time obtained excitatory connections are stronger and with bigger variance for the right LIP area (that corresponds to suppressed by reinforcement decision) while inhibitory connections achieved a little bit higher absolute values for the correct (left) LIP area.

In the case of reinforcement signal opposite to the perceptual decision Table III shows that excitatory connections to the suppressed by training left LIP area increase in comparison to previous case but remain the same for the three age groups while the absolute values of the inhibitory connections increased slightly with aging. Table IV demonstrates that when the reinforcement signal favors the right LIP area the excitatory connectivity increased with aging but has lower mean values and less variability than in the previous case. The inhibitory connections however remain the same for three age groups and achieved a little bit higher absolute values and variance.

These results might be explained with task-related connectivity between MST and LIP areas in our model. Since the excitatory connections are allowed only from MSTe templates to the corresponding to their focal points LIP areas and the rest of connections remain inhibitory, the reinforcement corresponding to perceptual decision increases inhibition from MSTe areas related to the wrong decision to both LIP areas while the opposite reinforcement tries to revert the strength of excitatory connections towards the one opposite to the
TABLE V

MEAN OF WEIGHTS OF CONNECTIONS FROM LGN TO TRN IN CASE OF REINFORCEMENT SIGNAL CORRESPONDING TO PERCEPTUAL DECISION (LEFT).

\begin{tabular}{|l|c|c|c|c|}
\hline Group & ON1 & ON2 & OFF1 & OFF2 \\
\hline Young & -0.99001 & -0.97580 & -0.86800 & -0.85067 \\
\hline Middle & -0.99126 & -0.97732 & -0.86824 & -0.85042 \\
\hline Elderly & -0.98803 & -0.97743 & -0.86915 & -0.85175 \\
\hline
\end{tabular}

TABLE VI

VARIANCE OF WEIGHTS OF CONNECTIONS FROM LGN TO TRN IN CASE OF REINFORCEMENT SIGNAL CORRESPONDING TO PERCEPTUAL DECISION (LEFT).

\begin{tabular}{|l|c|c|c|c|}
\hline Group & ON1 & ON2 & OFF1 & OFF2 \\
\hline Young & 0.00518 & 0.00444 & 0.00619 & 0.00796 \\
\hline Middle & 0.00514 & 0.00463 & 0.00625 & 0.00798 \\
\hline Elderly & 0.00528 & 0.00437 & 0.00640 & 0.00795 \\
\hline
\end{tabular}

perception decision.

Concerning the deep thalamic relay, in [14] we observed that only inhibitory feedback connections to LGN from TRN structure are subject to some changes so here we monitored only their changes. In contrast however to our previous results from STDP training of perceptual part of the model reported in [14], changes in case of reinforcement training reported here became visible after single presentation of teaching signal.

In case of reinforcement signal corresponding to perceptual decision (Tables $\mathrm{V}$ and VI) we observe slight decrease of inhibition with aging in connections only to first LGN layer having $\mathrm{ON}$ receptive fields $(\mathrm{ON} 1)$ and slight increase for the rest of LGN layers (ON2, OFF1, and OFF2).

In case of reinforcement opposite to the perceptual decision (Tables VII and VIII) we observed a tendency towards a decrease of inhibitory connectivity with aging to all LGN layers. Achieved in this case connection weights however are a little bit smaller in comparison with previous case of reinforcement training.

\section{TABLE VII}

MEAN OF WEIGHTS OF CONNECTIONS FROM LGN TO TRN IN CASE OF REINFORCEMENT SIGNAL OPPOSITE TO PERCEPTUAL DECISION (LEFT).

\begin{tabular}{|l|c|c|c|c|}
\hline Group & ON1 & ON2 & OFF1 & OFF2 \\
\hline Young & -0.99001 & -0.97830 & -0.87068 & -0.85187 \\
\hline Middle & -0.98981 & -0.97705 & -0.87082 & -0.84828 \\
\hline Elderly & -0.98722 & -0.97753 & -0.86884 & -0.84816 \\
\hline
\end{tabular}

TABLE VIII

VARIANCE OF WEIGHTS OF CONNECTIONS FROM LGN TO TRN IN CASE OF REINFORCEMENT SIGNAL OPPOSITE TO PERCEPTUAL DECISION (LEFT).

\begin{tabular}{|l|c|c|c|c|}
\hline Group & ON1 & ON2 & OFF1 & OFF2 \\
\hline Young & 0.00500 & 0.00440 & 0.00636 & 0.00835 \\
\hline Middle & 0.00493 & 0.00458 & 0.00633 & 0.00766 \\
\hline Elderly & 0.00522 & 0.00441 & 0.00663 & 0.00757 \\
\hline
\end{tabular}


The aging however increased the variances of connections to ON1 and OFF1 and decreased those of connections to ON2 and OFF2 in both considered cases of reinforcement signal.

In summary, the ageing effects in thalamic relay demonstrated predominantly increased inhibition in case of reinforcement signal corresponding to perceptual decision and decreased inhibition for the opposite reinforcement training. This might be explained by the fact that the reinforcement signal suppressing the perceptual decision tries to invert the overall model perceptual attitude while the reinforcement signal corresponding to the perceptual decision leads to agerelated differentiating in this structure positioned deep in the perceptual part of the model.

\section{CONCLUSIONS}

The model, presented here incorporates all basic structures in the human brain responsible for decision making based on dynamic visual information in tasks with eye movement response starting from the visual information encoding, preprocessing, information extraction and accumulation and saccade generation biased by subcortical structures (BG) in the presence of external reinforcement.

The adjustment of the model parameters in the dynamic (dopamine and STDP) synapses by feeding reinforcement signal reflecting specific characteristics of the human performance provides further insight into the complicate interactions between different brain structures and their modification in the process of learning, acting and aging.

A future application of our model will be to investigate by simulations the behaviour of the brain structures involved in visual information processing and decision making in case of deterioration in any of its layers, i.e. to perform in-sillico modelling of brain lesions or other degenerative brain processes. Comparison of such simulated behaviour with patients' performance in visual tasks can support early and noninvasive diagnosis of some deceases of human brain.

\section{REFERENCES}

[1] A. G. Barto, R. S. Sutton and C. W. Anderson, C.W., "Neuronlike adaptive elements that can solve difficult learning control problems," IEEE Trans. on Systems, Man, and Cybernetics, vol. 13 (5), 1983, pp. 834-846. DOI: 10.1109/TSMC.1983.6313077

[2] M. N. Shadlen and W. T. Newsome, "Motion perception: seeing and deciding," Proc. Natl. Acad. Sci. USA, vol. 93 (2), pp. 628-633, 1996. DOI: 10.1073/pnas.93.2.628

[3] D. M. Herz, B. A. Zavala, R. Bogacz and P. Brown, "Neural correlates of decision thresholds in the human subthalamic nucleus," Current Biology, vol. 26 (7), pp. 916-920, 2016. DOI: 10.1016/j.cub.2016.01.051

[4] K. Dunovan, B. Lynch, T. Molesworth and T. Verstynen, T., "Competing basal-ganglia pathways determine the difference between stopping and deciding not to go," eLife, vol. 4, Article number e08723, 2015. DOI: 10.7554/eLife.08723

[5] A. G. Barto, "Adaptive critics and the basal ganglia," in J. C. Houk, J. L. Davis and D. G. Beiser, Editors, Models of Information Processing in the Basal Ganglia, MIT Press, Cambridge, MA; 1995, pp. 215-232.

[6] D. Joel, Y. Niv and E. Ruppin, "Actor-critic models of the basal ganglia: new anatomical and computational perspectives," Neural Networks, vol. 15, pp. 535-547, 2002. DOI: 10.1016/S0893-6080(02)00047-3

[7] M. J. Frank, L. C. Seeberger and R. C. O'Reilly, "By carrot or by stick: cognitive reinforcement learning in Parkinsonism," Science, vol. 306 (5703), pp. 1940-1943, 2004. DOI: 10.1126/science.1102941
[8] R. Bogacz and T. Larsen, T., "Integration of reinforcement learning and optimal decision-making theories of the basal ganglia,", Neural Computation, vol. 23 (4), pp. 817-851, 2011. DOI: 10.1162/NECO_a_00103

[9] K. Dunovan and T. Verstynen, "Believer-Skeptic meets actor-critic: Rethinking the role of basal ganglia pathways during decision-making and reinforcement learning,", Frontiers in Neuroscience, vol. 10, Article number 106, 2016. DOI: 10.3389/fnins.2016.00106

[10] P. Koprinkova-Hristova and N. Bocheva, "Spike timing neural model of eye movement motor response with reinforcement learning," Lecture Notes in Computer Science, in press.

[11] J. Igarashi, O. Shounob, T. Fukai and H. Tsujino, "Real-time simulation of a spiking neural network model of the basal ganglia circuitry using general purpose computing on graphics processing units," Neural Networks, vol. 24, pp. 950-960, 2011. DOI: 10.1016/j.neunet.2011.06.008

[12] R. Krishnan, S. Ratnadurai, D. Subramanian, V. S. Chakravarthy and M. Rengaswamyd, "Modeling the role of basal ganglia in saccade generation: Is the indirect pathway the explorer?," Neural Networks, vol. 24, pp. 801-813, 2011. DOI: 10.1016/j.neunet.2011.06.002

[13] S. Kunkel et al.,"NEST 2.12.0," Zenodo, 2017. DOI: 10.5281/zenodo. 259534

[14] P. Koprinkova-Hristova, N. Bocheva, S. Nedelcheva, M. Stefanova, B. Genova, R. Kraleva and V. Kralev, "STDP plasticity in TRN within hierarchical spike timing model of visual information processing," IFIP Advances in Information and Communication Technology, vol. 583 IFIP, pp. 279-290, 2020. DOI: 10.1007/978-3-030-49161-1_24

[15] P. Koprinkova-Hristova, N. Bocheva, S. Nedelcheva and M. Stefanova, "Spike timing neural model of motion perception and decision making," Frontiers in Computational Neuroscience, vol. 13, Article number 20, 2019. DOI: $10.3389 /$ fncom.2019.00020

[16] P. Koprinkova-Hristova, N. Bocheva and S. Nedelcheva, "Investigation of feedback connections effect of a spike timing neural network model of early visual system, " in Innovations in Intelligent Systems and Applications (INISTA), Thessaloniki, Greece, 2018, DOI: 10.1109/INISTA.2018.8466292

[17] S. Nedelcheva and P. Koprinkova-Hristova, "Orientation selectivity tuning of a spike timing neural network model of the first layer of the human visual cortex," Studies in Computational Intelligence, vol. 793, pp. 291-303, 2019. DOI: 10.1007/978-3-319-97277-0_24

[18] T. W. Troyer, A. E. Krukowski, N. J. Priebe and K. D. Miller, "Contrast invariant orientation tuning in cat visual cortex: thalamocortical input tuning and correlation-based intracortical connectivity," J. Neurosci., vol. 18, pp. 5908-5927, 1998. DOI: 10.1523/jneurosci.18-15-05908.1998

[19] J. Kremkow, L. U. Perrinet, C. Monier, J.-M. Alonso, A. Aertsen, Y. Fregnac and G. S. Masson, "Push-pull receptive field organization and synaptic depression: Mechanisms for reliably encoding naturalistic stimuli in V1," Frontiers in Neural Circuits, vol. 10, Article number 37, 2016. DOI: 10.3389/fncir.2016.00037

[20] A. Casti, F. Hayot, Y. Xiao and E. Kaplan, "A simple model of retinaLGN transmission," J. Computational Neuroscience, vol. 24, pp. 235252, 2008. DOI: 10.1007/s10827-007-0053-7

[21] M. Ghodratia, S.-M. Khaligh-Razavic and S. R. Lehky, "Towards building a more complex view of the lateral geniculate nucleus: Recent advances in understanding its role," Progress in Neurobiology, vol. 156, pp. 214-255, 2017. DOI: 10.1016/j.pneurobio.2017.06.002

[22] P. Gleeson, R. Martinez and A. Davison, "Network models of V1," Open Source Brain, http://www.opensourcebrain.org/projects/111.

[23] S. Sadeh and S. Rotter, "Statistics and geometry of orientation selectivity in primary visual cortex," Biol. Cybern., vol. 108, pp. 631-653, 2014. DOI: $10.1007 / \mathrm{s} 00422-013-0576-0$

[24] M.-J. Escobar, G. S. Masson, T. Vieville and P. Kornprobst, "Action recognition using a bio-inspired feedforward spiking network," Int. J. Comput. Vis., vol. 82, pp. 284-301, 2009. DOI: $10.1007 / \mathrm{s} 11263-008-$ 0201-1

[25] O. W. Layton and B. R. Fajen, "Possible role for recurrent interactions between expansion and contraction cells in MSTd during self-motion perception in dynamic environments," Journal of Vision, vol. 17 (5), Article number 5, 2017. DOI: 10.1167/17.5.5

[26] W. Potjans, A. Morrison and M. Diesmann, "Enabling functional neural circuit simulations with distributed computing of neuromodulated plasticity," Front. in Comp. Neuroscience, vol. 4, 2010. DOI: 10.3389/fncom.2010.00141

[27] J. L. Plotkin and L. A. Goldberg, "Thinking outside the box (and arrow): Current themes in striatal dysfunction in movement disor- 
ders," The Neuroscientist, vol. 25 (4), pp. 359-379, 2019. DOI: $10.1177 / 1073858418807887$

[28] W. Wei, J. E. Rubin and X.-J. Wang, "Role of the indirect pathway of the basal ganglia in perceptual decision making," The Journal of Neuroscience, vol. 35 (9), pp. 4052-4064, 2015. DOI: 10.1523/JNEUROSCI.3611-14.2015

[29] H. Yan and J. Wang, "Quantification of motor network dynamics in Parkinson's disease by means of landscape and flux theory," PLoS ONE, vol. 12 (3), Article number e0174364, 2017. DOI: 10.1371/jour- nal.pone. 0174364

[30] M. Tsodyks, A. Uziel and H. Markram, "Synchrony generation in recurrent networks with frequency-dependent synapses," The Journal of Neuroscience, vol. 20 (1), pp. RC50, 2000. DOI: 10.1523/jneurosci.20$01-\mathrm{j} 0003.2000$

[31] N. Bocheva, B. Genova and M. Stefanova, "Drift diffusion modeling of response time in heading estimation based on motion and form cues," Int. J. of Biology and Biomedical Engineering, vol. 12, pp. 75-83, 2018. 